\title{
A coordination framework based on the Sociology of the Organized Action
}

\author{
C. Sibertin-Blanc, F. Amblard, M. Mailliard \\ IRIT - Université de Toulouse 1 \\ 21, allées de Brienne, 31042 Toulouse Cedex - France

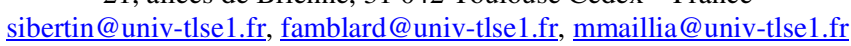

\begin{abstract}
This paper proposes a basis to design coordination models in multiagent systems. This proposal is based on the exploitation of an in-depth exploration of a well-experienced sociological theory, the Sociology of Organized Action. This theory intends to discover the functioning of an organization beyond its formal rules, especially how social actors define the organization that in return rules their behaviours, and which are the mechanisms they use to regulate their interactions. We then first present the concepts developed by this theory to understand the strategic aspects of the actors' behaviours in an organized actions framework. Then we introduce a metamodel that allows us to describe the structure of Concrete Action Systems and how social actors handle its elements. A classical case study is used to illustrate the approach.
\end{abstract}

\section{Introduction}

Agents' coordination mechanisms within organizations pose new demands compared to traditional Multi-Agent Systems models, such as the integration of organizational and individual objectives with possible problems of compatibility, the dynamic adaptation of agents' behaviours to organizational changes, or conversely the way agents' behaviour could lead to organizational changes. As systems grow to include increasing number and heterogeneity of agents, the coordination has to be improved in order to consider both the agent-centric, as well as the organizationcentric views. However, formal theories, tools and methodologies are still very much in short supply. Even if an externally designed organizational structure is necessary as a coordination device to achieve global social order, there is a possibly inefficient and ineffective tension between such imposed constraints and the agents' autonomous behaviour.

In order to enrich this field, we think that a controlled metaphor based upon well founded sociological theories could enable to design high-quality models for coordination in agents' organizations. Some works similar to the Agent-Group-Role paradigm [1] showed the limits of approaches, which, inspired from metaphors with the fields of psychology or cognitive sciences, are exclusively centred on the structure and the abilities of the agents (cf. architectures like Belief-Desire-Intention [2]). The focus on the organizational level is actually at play in many works in Multi-Agent or 
Component-based Systems. Our work follows the line of works like the ones of Malone and Crowston [3], of Castelfranchi [4] or Hermann [5], who research in the sociology a pertinent and well-funded metaphor for a coordination model allowing to root the definition of the organizational level in MAS. The emergent coordination mechanisms of the Sociology of the Organized Action [6], which are based upon very abstract concepts, are susceptible to serve as a suggestive source of inspiration and to be used in several application domains.

We then first present the sociological theory that is the basis for our proposal, namely the Sociology of the Organized Action (SOA), insisting on the major concepts we retained to build up a meta-model of this theory. We therefore present in the third section our framework based on the concepts of Actors and Resources-Relations, the things in the Actors' organizational environment they use to establish control and dependency links between them - in fact power relationships. This is the static aspects of the meta-model, e.g. the objects present in the model as well as the objects manipulated by the actors, allowing to describe the structure of a social system. The fourth section presents the dynamics aspect of the model. We focus explicitly on the distinction between functional and structural dimensions of the actors' actions, and how we do manage this distinction in the current version of the SocLab simulation environment. Finally in a last part, we exemplify the approach on a concrete system that is a classical example taken from the literature in the Sociology of the Organized Action. It enables us to illustrate our proposed model of coordination as well as pointing certain limits of our approach that are currently under investigation.

\section{The Sociology of the Organized Action}

The Sociology of the Organized Action (SOA) aims at discovering the real functioning of an organization beyond its formal rules. The Concrete Action Systems (CAS) under study, for instance a firm or a local political system, are composed by «numerous differentiated actors interacting in a non-trivial way among each others » [6]. Moreover, these actors are engaged durably in the achievement of the organization's objectives. A CAS is an interaction context precisely delimitated which structures the cooperation among a set of actors. This structure is admittedly constraining but without removing all freedom in the way of acting. It deals then with relatively structured relational contexts and the SOA does not aim to relate spontaneous effects like crowd behaviours or riots [7]. If the sociology of the organized action inherits the sociology of organizations [8], its application scope spreads to all kind of «organized» action whatever its level of codification or formalization. The SOA focuses on regulation phenomena which ensure at the same time the evolution of such systems and their relative stability.

The SOA is built upon the idea that organizations are social constructs actualised by and within the relationships among the organizational actors. Moreover, this theory classically assumes that actors behave strategically following the bounded rationality paradigm [9].

Each actor's behaviour is then neither totally conditioned by the organisational rules that constrain him, neither it is by pure individual or emotional factors. This 
behaviour is strategic. Then the induced actions aim at realizing some objectives, would it be conscious or not. Beyond the achievement of both his own objectives and those given by the organization's formal norms, each actor aims, as a meta-objective, at having enough power to be able to preserve or increase his autonomy and his action capacity within the organization.

This power results from the control of one or several uncertainty zones that enable him to behave in an unpredictable way and consequently to decide, more or less, the exchange rules concerning his relations with others. Each uncertainty zone is a resource for the action, and thus at the same time a constraint and an opportunity. The interactions among actors regulate those power relationships and as a consequence transform the related uncertainty zones, their control as well as their relevance, and then the rules of the social game. The four main uncertainty zones identified by the theory and implemented in the frame of power relationships of a CAS are based upon: competence or expertise; the control of the interactions with the environment of the organization; the control of the internal communication; and the knowledge and the proper use of the organization's norms and rules.

To summarize, the Sociology of Organized Action is a theory of the action that explains the running of organizational processes, taking into account the double dependency between the actor and the system, by using the concepts of bounded rationality, power relationships, uncertainty zones and the one of concrete action system. This theory and the related concepts serve as a theoretical basis as well as an analysis grid to study many cases: the introduction of the automation in a traditional firm or the decision-making process during the crisis of the Cuba's missiles [10,6]. Interested readers can refer to [8] for a detailed analysis of ten case studies.

\section{Proposed meta-model}

A formalization of the SOA leads to consider that constitutive elements of a concrete action system are of the three different types shown in Fig. 1: the Actor, the Relation and the Resource. We adopt therefore the term Resource rather than Uncertainty Zone from the SOA terminology because every uncertainty zone is a resource, and its constitutive property is less the uncertainty on the behaviour of its controller than the existence of actors who need this resource while they don't control the conditions of its use. 


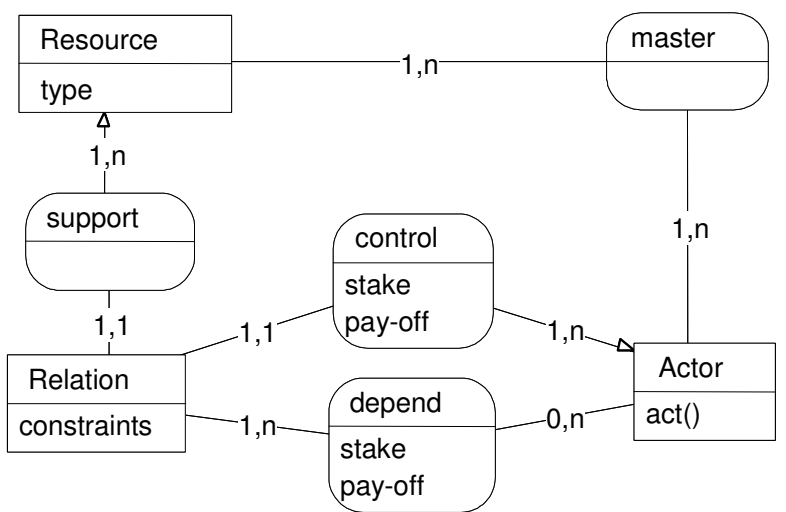

Fig. 1. Model of the structure of the CAS (using the Entity/Association formalism).

To describe briefly the figure 1, a Resource can be the support of one or more Relations associated to Actors who are linked to it, either because they control the Relation or they depend on it. Each actor puts stakes and receives in return a pay-off for each one of the Relations he is implied in. The actor who masters a Resource (by the mean of a Relation he controls) decides of the distribution of the pay-offs among the actors who depend on this Relation.

\subsection{Actor, Resource and Relation}

The Resources of a CAS are the things necessary for the organized action, their availability being required in order to make some action.

Every Resource is mastered by one or more Actors who decide about its availability and therefore influence the action capability of the Actors who need it. Each Resource leads to the creation of one or several Relations. A Relation corresponds to a certain type of transaction, or bargaining concerning the use of this Resource. A Relation is unbalanced as a unique Actor (among the ones who master the Resource) controls this Relation while other Actors depend on this Relation because they need this Resource to achieve their goals. The controller of the Relation determines the conditions of the access to the Resource and controls the possibility for the depending Actors to perform the actions linked to their objectives.

Every Actor masters one or more Resources and then possesses some freedom to act that he exerts by means of the Resources he controls. As a result, the SOA denies the status of social actor to a person who would not master any Resource. The concepts of Resource and Actor are then defined one from the other: a Resource is such only if some Actor depends on it in order to act; conversely, a social actor is somebody who controls at least one Resource. 


\subsection{Stakes and pay-offs}

Each Actor distributes his stakes on each one of the Relations he participates to, either by controlling them or depending on them. He makes this repartition depending on the importance of the Resource in regards to his objectives. The more necessary the use of the Resource to achieve an important objective, the higher the stake he places on this Relation. Figure 2 shows how to integrate explicitly the concept of objective in the model of a CAS. For a given Actor and a given Relation, the value of the stake property in the associations control and depend is determined by the value of the properties importance and necessity. This repartition of an Actor's stakes is in proportion with the impact of these objectives on his behaviour. For the understanding of the functioning of a CAS, the very identification of the objectives of an Actor does not matter, much more important is what they lead the Actor to do. The stakes enable to link causally the Actor's behaviour with his objectives. The stakes take their value on a qualitative scale like: null, negligible, ..., important, ..., vital and could be therefore translated on a numerical scale we took for the example below between -10 and +10 .

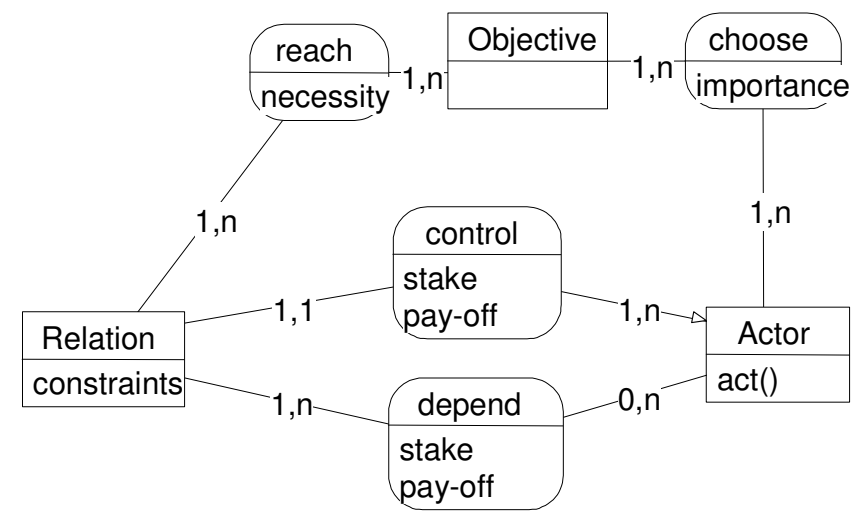

Fig. 2. Place of the Objective concept in the formalisation of the CAS.

The Actor controlling a Relation is the one who determines the exchange rules, that is the conditions governing transactions concerning the access to the Resource. We prefer the term pay-off, more concise, which evocates the result of the transaction rather than the modalities of its processing. The pay-off corresponds to the quality of the Resource availability; more or better the usability of the Resource by an Actor, higher his pay-off for this Relation. The distribution criterion of the pay-offs between the different participants of a Relation is specific to each Relation. We are expecting Relations where the pay-offs are a «zero sum game »: if the usability of the Resource is good for some actors, it will be as bad for the others. Other Relations for which the pay-offs of the controller and depending Actors vary in the same direction could be qualified of win-win, or loose-loose whether the pay-offs are favourable or not. These pay-offs can take their value on a scale like: awful, ..., bad, ..., neutral, ..., good, ..., 
optimal and therefore could be translated on a numerical scale we took between -10 and +10 for the example given below.

\subsection{The constraints on a Relation}

We now have to give the meaning of the constraints property of Relations. The Actor who controls a Relation has not the possibility to give any value to the pay-off property of the participating Actors. He has to respect the « rules of the game » that regulate the interactions among the actors of the organization and determine the range of value he may attribute to the pay-offs. These constraints originate either from formal rules of the organization or from rules imposed by the environment, either from technical or feasibility restrictions that result from the very nature of the Resource, or from social norms that determine the socially acceptable behaviours. (The sociology of organized action does not include such a classification of constraints, but a deeper analysis of the various types of Resources and associated constraints could ease the modelling of the structure of CASs). In addition, we have to deal with the fact mentioned in the previous section that the values of pay-offs attributed to the different Actors are in relation. So we propose to formalize the constraints associated to a Relation as the following items:

- $\quad$ two boundary values $b \_\min$ and $b \_\max$, such that $-1 \leq b \_\min <b \_\max \leq 1$;

- $\quad$ for each actor $A$ participant in the relation, a function

$$
\text { Effect }_{A}:[-1,1] \longrightarrow[-10,10] \text {. }
$$

The interval $[-1,1]$ correspond to the theoretical space of choice for an Actor that would have a full control upon the Relation: choosing a value within this interval corresponds to choosing how he manages the relation and so what kind of access he gives to other Actors concerned by the relation. Then $\operatorname{Effect}_{A}(\alpha)$ will be the value of the balance for an Actor $A$ resulting from the choice (by the controller Actor) of a value $\alpha \in[-1,1]$. It is clear that any number can be used instead of -1 and +1 as the boundaries of the space of choice, and only the relationships between the different functions Effect $_{X}, X$ being the Actors participant in the Relation, is of matter. (In order to chose an interval $[a, b]$ as the space of choice instead of $[-1,1]$, you just have to compose the Effect $_{X}$ function with the function $x: \longrightarrow 2 /(b-a) * x-(a+b) /(b-a)$; The convenience of the $[-1,1]$ interval as spaces of choice relies upon its similarity with the range of pay-off values, that is $[-10,10])$.

As for the $b \_\min$ and $b \_\max$ boundaries, they are intended to deal with the fact that the Actor controlling the relation is possibly in a situation where he cannot select whatever value in the space of choice. For any reason, his effective space of choice is more limited and then he can only chose a value within the [b_min, $b \_$max $]$interval. So the range of this interval (that is the number $b \_$max $-b \_$min) is a measure of the extent of the control on the Relation by the controller Actor.

This formalization enables to quantify the influence that the Actor controlling a Relation $\mathrm{R}$ is able to exert on another Actor $A$ participating in the Relation. If you consider:

$$
\text { influence }_{R}(A)=\max \left\{\operatorname{Effect}_{A}(\alpha)-\operatorname{Effect}_{A}(\beta) ; \alpha, \beta \in\left[b \_ \text {min, } b \_ \text {max }\right]\right\},
$$


you have the maximum difference between the balances that he can attribute to Actor $A$, that is the greatest amplitude of the effect of his choice in the management of the Relation.

The global influence of the Actor controlling the Relation $R$ can then be defined as the greatest influence subjected by one of the Actors:

influence $_{R}=\max \left\{\right.$ influence $_{R}(A) ;$ A Actor concerned by the relation $R$ \}.

Indeed, the Actor subject to the greatest influence will behave accordingly and thus pass the effect of this influence to other Actors. So, one can consider that this high level of influence is the one that will spread over the whole organization.

The following quantity

$\max _{\operatorname{Effect}}(\alpha)-\operatorname{Effect}_{A}(\beta) ; A$ Actor concerned by the relation $R, \alpha$ and $\left.\beta \in[-1,1]\right\}$

may lead to consider the strength of the Relation as a tool for the power. Then the influence $_{R}$ of the controller of Relation $R$ is a weighting of this strength by his level of mastering of $R$ (that is the range of his effective space of choice), and thus corresponds to the effective usability of this relation as a support for power.

\section{Actors' behaviours and model dynamics}

The modelling formalism we exposed enables to distinguish, within a CAS, what corresponds to its structure - its constitutive elements and their relations -, and what corresponds to its state which change follows the achievement of the system goals. The CAS's structure can be described in terms of Actors, Relations based on Resources, controls and stakes placed on these Relations; concerning the CAS's state, it can be described for each actor using the pay-offs on the relations he participates to, that is the available means for action.

\subsection{Structural and functional dimensions of the actors' behaviour}

This allows to distinguish two dimensions for the actions of an actor who searches to comfort his power: a structural dimension which acts on the system structure, and a functional dimension which acts on the system state (Cf. Figure 3). The action's structural dimension contributes to the building of the CAS organization, to the establishment of the social game rules and then consists in, following our formalization, acting on the elements which constitutes its structure: the Resources, the Relations, the constraints and the stakes. Concerning the action functional dimension of an actor, it is the one which insures the regular functioning of the system and makes its state to evolve in a synchronic way. It participates in the achievement of the actor's immediate objectives. This functional dimension of Actors' actions complies with the current rules of the game, without regard for possible changes concerning the mission and objectives (i.e. the stakes) or the means for action (i.e. the relations and the associated constraints). In the behaviour of a human being social actor, each concrete action comprises a structural and a functional component in a proportion specific to the circumstances of the action achievement. When modelling a CAS, we are not trying to account for the practical modalities of the actions, instead 
we only focus on their effects. These effects on the structure and on the state of a CAS being disjoins, we have the possibility, concerning simulation issues, to model the actor's actions by the mean of specific mechanisms for each one of these two dimensions.

Within the structural dimension of actors' behaviour, actions deal with the Resources, the Relations, the constraints and the stakes. Concerning Resources, an Actor can introduce a new Resource with an associated Relation that he will master by using his proper capacities, or rather introduce a new Relation based on a Resource that he is yet mastering. An Actor can also neutralize the possibility of another Actor to control a relation, for instance by giving an open access to the Resource or conversely to make it definitely inaccessible whatever the circumstances. Another possible structural action is to transform a Resource in such a way that some $\operatorname{Effect}_{X}()$ functions become modified. Concerning the constraints which apply on the pay-offs of Relations, an Actor can decrease the severity of a relation he controls by enlarging the effective space of choice (decreasing $b \_$min or increasing $b \_$max), or conversely reinforce the severity of constraints applied to a relation controlled by another actor. Finally, concerning the stakes, actors can move their own stakes to reinforce their autonomy, but also and over all they can influence other actors in the distribution of their stakes.

Within the functional dimension of the actor's behaviour, every action consists in exerting control on a mastered relation, e.g. manipulating the pay-offs value attributed to the participating actors, while staying inside the limits imposed by the constraints of this relation. This manipulation can be absolute, then it modifies the pay-offs value without care to their current value, or relative if it increases or decreases regularly this value. This latter case corresponds to a continuous management of the control, without sudden shift, which seems to be the norm in many social activities.

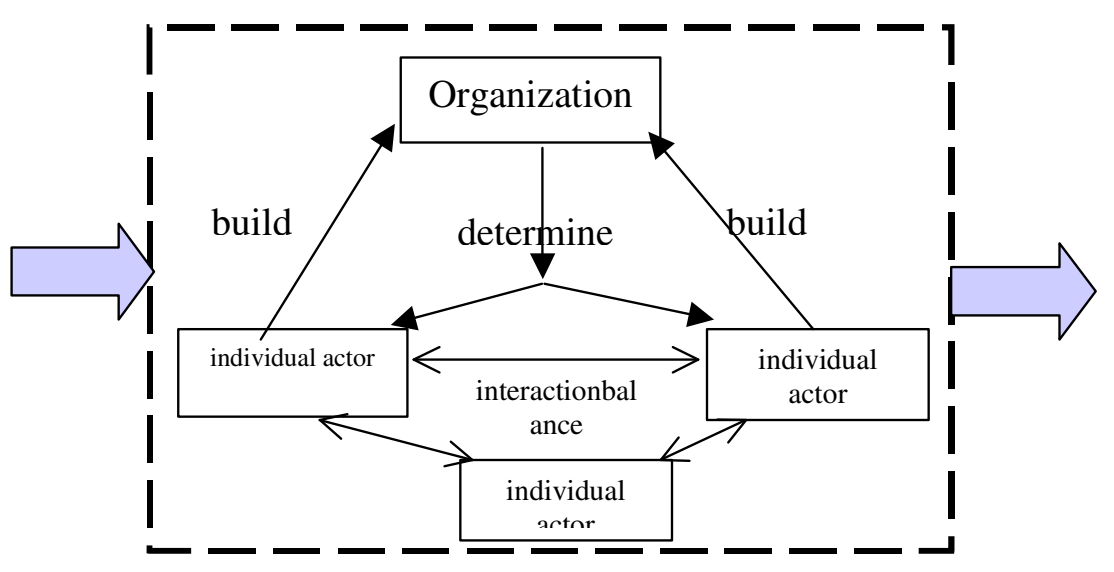


Fig. 3. The structural behaviour of Actors builds the organisation that in return constraints their functional behaviour.

\subsection{Actor's satisfaction and strategic behaviour}

The distribution of pay-offs and stakes on numerical scales enables, applying simple operations, to aggregate those values in synthetic and significant values. One can graduate the stakes on a scale null $=0$, negligible $=1$, important $=5$, vital $=10$, and the pay-offs with the correspondence $a w f u l=-10$, bad $=-5$, neutral $=0$, good $=5$, optimal $=10$. As evidence, these numerical values do not correspond to something; they just enable to perform comparison among them. To do so, we have to normalize the sum of the actors' stakes and then attribute the same amount of stakes to each actor for him to distribute on the relations he participates to. This normalization comes down to grant the same investment to each actor, the same possibility of personal implication in the social relations game.

It is therefore possible to quantify several concepts of the SOA by using numerical values belonging to the same scales in order to compare them. For instance, the relevance of a Resource could be estimated as the sum of the stakes placed by the whole population of actors on the Relation supported by the Resource, as those stakes reflect the importance of these Relations for the actors. The Actor's power can be also estimated as the sum, over all the relations he controls, of a combination between the relevance of this Relation and the influence of this Relation. The actor's autonomy can be evaluated as the sum of the stakes he places on the Relations he controls. It corresponds to the possibility to achieve his objectives independently from other actors, the actor's dependency being evaluated conversely as the sum of the stakes he places on the Relations he depends on. Other notions like the actor's power on another one or the dependency network among actors could be defined also.

A particularly significant value is, for each actor, the sum on the whole set of Relation he is involved in, of a combination between his stake and the resulting payoff he receive. We name this value the actor's satisfaction (rather than utility because it is more linked to a bounded rationality context). It expresses the possibility for an actor to access to the resources he needs in order to achieve his objectives, and then the means available for him to achieve these objectives. A linear version consists in considering the sum, on every relation he is involved in, of the stake by the pay-off:

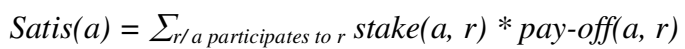

To obtain or preserve a high level for this satisfaction is a meta-objective for every actor, as this level determines his possibility to achieve his concrete objectives.

The strategic characteristic of an actor's behaviour leads him, by definition, to aim and achieve his objectives and then to obtain an acceptable value (if not the optimum) for his satisfaction. The rationality hypothesis implies to build this behaviour on the standard three steps cycle:

1. perception of its own state and of the environment;

2. selection of an action to perform, according to its expected effect on the gap between the current and the goal state; 
3. execution of this action.

We have implemented a simulation environment, SocLab [11], that allows to describe the structure of a CAS according to the meta-model introduced in section 3 and to simulate the functional dimension of Actors' behaviours, that is the mutual adjustment of the payoffs they give the ones to the others. This "social behaviour engine" uses the classifiers mechanism [12] for the selection of the action; it is based upon the learning of behavioural rules by test-errors and reinforcement of the rules depending on the results they produce. This approach presents two advantages compared to a cognitive approach [13]: we only need a global model of the CAS, where the cognitive approach requires to explicit the representation of the CAS by each one of the actors; it brings also little hypothesis on the required abilities to be an actor within a CAS, and it not need to explicit the rules governing the social behaviour of the actor.

\section{The Trouville case}

To illustrate how the SOA analyses a system of concrete action and how we formalize this analysis, we will consider a classical example from the strategic analysis (the other name for the SOA) [14]. The Travel-tours firm is a tour operator having two agencies, TRO1 and TRO2, situated in Trouville. These last months, the results of the TRO1 agency increases, as the ones of TRO2 agency stay stable, or even decrease. The regional director decided to reward the TRO1 agency for its merits. He proposes then to regularize the situation of Agnès, the secretary of the agency and to affect her exclusively to TRO1. As she is temporary employed for several months, and even if she is attached to TRO1, she works half time in each one of the agencies and this obliges her to move between her two jobs.

Either Agnès or the TRO1 agency's director, Paul, should be glad with this proposal. Agnès will have a more permanent work contract and will be relieved to split her work in two parts, while Paul will have a full-time secretary in his agency. But each one of them refuses vigorously the proposal. How to understand this matter of fact? The strategic analysis by identifying the uncertainty zones shows that both of them are rationally right to be opposed to this organizational change, because it would decrease their respective power. Indeed, a more attentive analysis of the case reveals that:

- The TRO2 agency is more inventive than TRO1 in designing travel packages, while the TRO1 agency includes a very efficient commercial staff; so, the TRO1 agency director takes full advantage of finalizing the TRO2's ideas.

- For personal reasons, to get a steady job is not a short-time objective of the secretary. On the other hand, she is very cool in her working relations with the other employees of TRO1, and she greatly appreciates this feature of her job

Thus the situation shift would increase the control of the director on the secretary's activities (that is what she does not want) and as a counterpart the director would loose the information given by the secretary on TRO2 (that is what he does not want). 


\subsection{Model of the concrete action system}

The purpose of the analysis is to understand the behaviour of the director and the secretary, so both of them are Actors to be considered, and it appears that other employees of the TRO1 or TRO2 agencies do not play a role is this affair. Concerning the uncertainty zones or Resources, Information about TRO2 is the one mastered by the secretary while the director masters the secretary's job. This latter Resource gives raise to two different Relations between the director and the secretary: the stability of the job and the content of the work she has to achieve. Table 1 shows the values given to the different parameters of the model. The value of stakes results straight from the observations below about the situation of the director and the secretary. Both of them have ten points to distribute over the three Relations, and the relevance of each Relation is just the sum of the stakes.

The value of the $b \_\min$ and $b \_\max$ bounds and the definition of the Effect functions require more explanations. Concerning the stability of the job, the director has only a partial mastering of this Relation; on one side he may renew the contract of the secretary each week without discussion, but he may not set a firm contract to on his own, only the regional director can do this, so $b \_\max \approx 0.4$; on the other side, he has to respect the job legislation, to justify his decision to the regional director, and to account for the reaction of other employees in case of clear unfairness, so $b \_$min $\approx$ 0.4. Having a steady job produces a full effect for the secretary and thus Effect $_{\text {secretary }}(1)=10$, Effect $_{\text {secretary }}(-1)=-10,-10$ and 10 being the extreme values of a balance. As for the director, his worry about this job is in proportion with the stability of this job, but this worry is quite low.

Concerning the content of the job, the agency director has a larger room of manoeuvre. We consider positive values in the space of choice as a strict control on the quantity and the quality of the secretary's work and how she organizes this work, and negative values as the lack of such a control. The $b \_$min $\approx-0.3$ value results from a high concern of the director for having friendly relationships with the employees; nevertheless, he has to ensure the production of the agency and thus to have a look on the work achieved by each employee, so the $b \_$max $\approx 0.7$ value. The effect on the secretary is in proportion with the level of control, because the convenience of any employee is to suffer a low level of control of his/her work. As for the director, the proposed effect function is based upon the ideas that any excess or lack of control could bring some difficulties and that his interest is to exercise a moderate control.

For the information about the TRO2 agency Relation, positive values in the space of choice correspond to give information about the projects of TRO2, negative values to give false information, and the zero to give no information. The $b \_$min and $b \_$max proposed values correspond to the amount of information on TRO2 that the secretary can obtain and make to be credible by the TRO1's director. The effect function for the director models his full use of this information; as for the secretary, her own tranquillity would be to give no information, neither real nor false.

Table 1. Parameters of a formal model for the Travel-tours case study

\begin{tabular}{c|c|c|}
\hline $\begin{array}{c}\text { stability of } \\
\text { the job }\end{array}$ & $\begin{array}{c}\text { content of } \\
\text { the job }\end{array}$ & $\begin{array}{c}\text { information } \\
\text { about TRO2 }\end{array}$ \\
\hline
\end{tabular}




\begin{tabular}{|c|c|c|c|c|}
\hline \multicolumn{2}{|c|}{ Controller Actor } & Director & Director & Secretary \\
\hline \multirow{2}{*}{ stake } & Director & 1 & 2 & 7 \\
\cline { 2 - 5 } & Secretary & 2 & 7 & 1 \\
\hline \multicolumn{2}{|c|}{ relevance } & 3 & 9 & 8 \\
\hline \multirow{2}{*}{ Effect } & Director & $3 * \mathrm{x}$ & $-3 * \mathrm{x}^{2}$ & $10 * \mathrm{x}$ \\
\cline { 2 - 5 } & Secretary & $10 * \mathrm{x}$ & $7 * \mathrm{x}$ & $-\left.2 *\right|_{\mathrm{x}} \mid$ \\
\hline \multicolumn{2}{|c|}{ b_min , b_max } & $-0.4,0.4$ & $-0.4,0.4$ & $-0.3,0.7$ \\
\hline \multicolumn{2}{|c|}{ influence } & $0.8 * 10=8$ & $1 * 7=7$ & $1.1 * 10=11$ \\
\hline \multicolumn{2}{|r|}{} & & \\
\hline
\end{tabular}

\subsection{Behaviour of Actors}

Table 2. Satisfaction and power of Actors

\begin{tabular}{|l|c|c|c|c|c|}
\cline { 2 - 6 } \multicolumn{1}{c|}{} & $\begin{array}{c}\text { Secretary's } \\
\text { optimum } \\
\text { satisfaction }\end{array}$ & $\begin{array}{c}\text { Director's } \\
\text { optimum } \\
\text { satisfaction }\end{array}$ & $\begin{array}{c}\text { Pareto } \\
\text { optimum } \\
\text { satisfaction }\end{array}$ & $\begin{array}{c}\text { auton } \\
\text { omy }\end{array}$ & power \\
\hline Director & $-1,7$ & $\mathbf{5 7 , 2}$ & 54,3 & 3 & 87 \\
\hline Secretary & $\mathbf{4 2 , 3}$ & 6,4 & 40,7 & 1 & 88 \\
\hline global satisfaction & 40,5 & 63,5 & $\mathbf{9 5}$ & & \\
\hline stability of the job & 0,4 & 0,4 & 0,4 & & \\
\hline content of the job & 0,7 & 0 & 0,7 & & \\
\hline info on TRO2 & 0 & 0,8 & 0,8 & & \\
\hline
\end{tabular}

Table 2 shows three typical states of the system resulting from the values given in table 1 . The cases where the secretary or the director have their maximum satisfaction are not socially feasible. Indeed, the last three rows of the table show the choices made by the Relation's controller that yield to the corresponding satisfaction; considering the Secretary's optimum satisfaction as an example, the director has no reason to be especially indulgent with her if she does not bring a specific advantage to the agency. More generally, no Actor will accept to relinquish the power given by the control of a Relation if this renouncement leads to a situation that is too far from an acceptable satisfaction. We observe that the maximal global satisfaction, that is the Pareto optimum, is reached with each Actor having the most cooperative behavior; but this fact is specific to the Travel-tour case study. Figures 4 and 5 show simulations of this case study with the SocLab environment. In all these simulations, the satisfactions of both Actors stabilize at a level that is near the Pareto optimum.

All these numerical results must be considered very carefully if one intends to give them an effective social interpretation. First, the scales of values are arbitrary, so that each value considered in isolation has no meaning; only the relative values of parameters make sense, and the results are given only for comparison. Second, the gap between two values must be important - e.g. 20 or 30 per cent - to be considered as meaningful. Indeed, the values of the parameters resulting from the sociological 
analysis are rough in nature. Moreover, the formulas proposed to evaluate the power or satisfaction of Actors are not the result of a formal argument; they are grounded in a firm sociological theory but intend only to be an approximation of these concepts. Finally, we agree with a bounded rationality framework that considers affairs from a qualitative point of view rather than a quantitative one.

Concerning the Travel-tour case, a sensitivity analysis shows that the model summed up in table 1 is quite robust. But the best use of the numeric values introduced by our meta-model would be to process and interpret them within a fuzzy calculus [15].

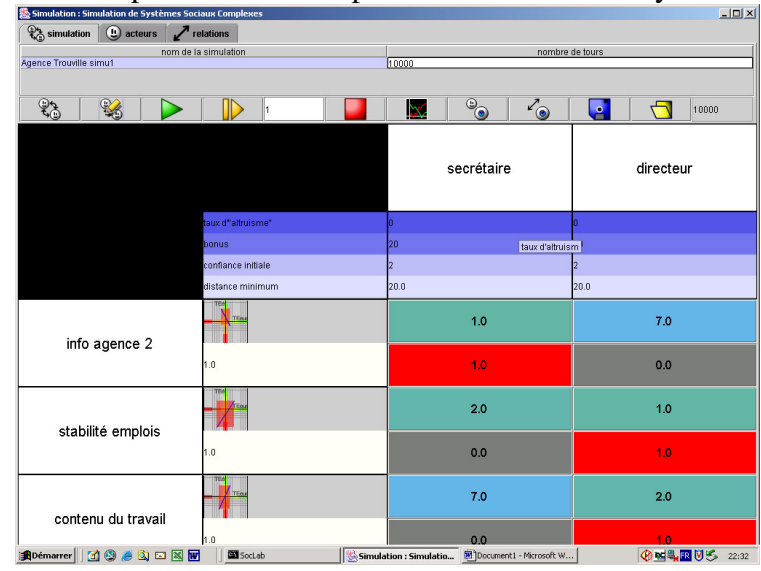

Fig. 4. Matrix expressing the stakes and controls on the relations.

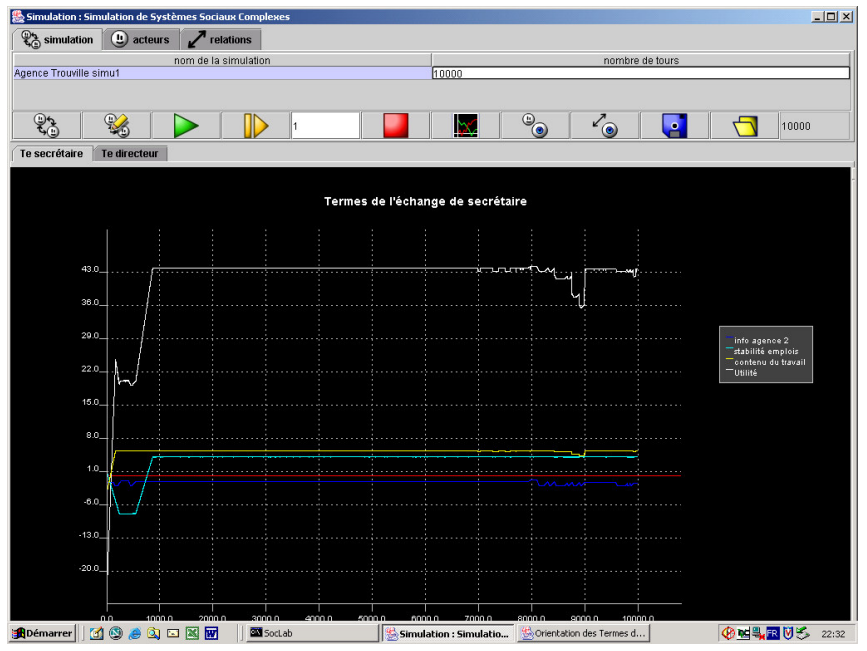


Fig. 5. Evolutions of the satisfaction (sum of the balances weighted by the stakes) of the secretary during one simulation.

\section{Conclusion}

From the viewpoint of the Sociology, this project could appreciably transform both the practice and the teaching of the SOA by the possibilities offered by a tool which objectifies the attempts and results of the sociological analysis. Such an attempt to formalize a inherently discursive theory goes with interrogation about this theory; so this project has already proved to impact the theoretical corpus of the SOA [17], by the mean of the researches that could be done from a virtual experimental framework, a radically new approach in sociology [16]. Despite its notoriety and its generalized teaching in France, the Strategic Analysis had never been taken as a subject for modelling.

Several questions are still open in order for this model to be fully operational, either for sociologist, or for a model of coordination to define the organisational level in MAS, or for providing virtual creatures with a plausible human-like behaviour. Among these questions we can cite the following. Coalition-like actors, who have their own stakes, based on the objective of the coalition, but whose satisfaction results from the building of the members of this coalition; the resources dynamics: how to characterize resources and relations which can be removed or conversely introduced in a CAS; what are the circumstances that lead an Actor to try to change the structure of a CAS, what kind of change he will chose, and what are the means he could you to achieve this change. This last question, which nothing else than the auto-evolution of social systems, is probably one of the most difficult, but also one of the most interesting if we focus on the expressive power of this coordination model.

\section{References}

1. Ferber, J., Gutknecht, O.: A Meta-Model for the Analysis and Design of Organizations in Multi-Agent Systems. Proceedings of the 3rd International Conference on Multi-Agents Systems (ICMAS), IEEE CS Press (1998).

2. Cohen, P.R., Levesque, H.J. : Intention is Choice with Commitment. Artificial Intelligence vol.42, (1990) 213-261.

3. Malone, T.W., Crowston, K. : The interdisciplinary study of coordination. ACM Computing Survey 26, 1, (1994).

4. Castelfranchi, C.: Modelling social action for AI Agents. Artificial Intelligence, 103, 157182, (1998).

5. Hermann, T., Jahnke, I., Loser K-U.: The Role Concept as a Basis for Designing Community Systems . In Cooperative Systems Design, M. Zacklad et al. (Eds), IOS Press, (2004).

6. Crozier, M., Friedberg, E. : L'acteur et le système : Les contraintes de l'action collective. Seuil (1977). 
7. Granovetter, M.: Threshold models of collective behavior. American Journal of Sociology, vol. 83, 1360-1380 (1978).

8. Bernoux, P.: La sociologie des organisations, Seuil, (1985).

9. Simon, H.: The sciences of the artificial, MIT Press, $3^{\text {rd }}$ edition (1996).

10.Crozier, M. (1962)

11.Mailliard, M., Audras, S., Casula, M. : Multi Agents Systems based on Classifiers for the Simulation of Concrete Action Systems. In Proceedings of the 1st EUropean Workshop on Multi-Agent Systems (EUMAS), Oxford University, (2003).

12.Holland, J., Booker, L.B., Colombetti, M., Dorigo, M., Godberg, D.E., Forrest, S., Riolo, R., Smith, R.E., Lanzi, P.L., Soltzmann, W., Wilson, S.W.: What Is a Learning Classifier System? LCS'99, LNAI 1813, 3-32 (2000).

13.Sichman, J., Conte, R., Demazeau Y., Castelfranchi, C.: Reasonning about others using dependence networks. Proc. of the 3ème italian Workshop on Distributed Artificial Intelligence, Rome, (1993).

$\begin{array}{lllll}\text { 14.Smets } & \text { P.: } & \text { L'agence } & \text { Travel-Tours } & \text { (Trouville). }\end{array}$ http://homepages.ulb.ac.be/ psmets1/travel.pdf.

15.Slowinski, R., Teghem, J.: Optimization models unsing fuzzy sets and possibility theory. Theory and decision library; Kluwer Academic Publishers, (1990).

16.Gilbert, N., Troitzsch, K.: Simulation for the social scientist. Open University Press, Londres, (1999).

17.Sibertin-Blanc C., Roggéro, P.: Pour une formalisation de la Sociologie de l'Action Organisée. Communication au XVII ${ }^{\text {ème }}$ congrès international des sociologues de langue française, 5-9 juillet 2004, Tours (2004). 